\title{
Therapeutic Acupunctural Resonance II: New Discoveries That Justify the Outcomes of This New Therapeutic Modality
}

\author{
Adrián ÁngelInchauspe $\mathbf{1}^{1,2,3,4,5,6,7,8,9,10}$ \\ ${ }^{1}$ Scientific Department, Argentine Acupuncture Society, Buenos Aires, Argentina \\ ${ }^{2}$ Argentine Resuscitation Council, City name, Country name \\ ${ }^{3}$ Research Department, HINEA and “Hospital Interzonal Neuropsiquiátrico 'Dr. Alejandro Korn””, La Plata \\ Argentina \\ ${ }^{4}$ Buenos Aires, Argentina \\ ${ }^{5}$ School of Medical Sciences, National University of La Plata, Buenos Aires, Argentina \\ ${ }^{6}$ National Delegate (Argentina) to the 9th International Brain Injury Association Congress, Edinburgh, UK \\ ${ }^{7}$ World Journal of Critical Care Medicine, Pleasanton, CA, USA \\ ${ }^{8}$ Frontiers of Clinical Pharmacology Research and Outcomes Journal, Lausanne, Switzerland \\ ${ }^{9}$ PCS World Cardiothoracic-Renal Disease, Chongqing, China \\ ${ }^{10}$ Journal of Acute Disease (JAD), Hainan Medicine University, Haikou, China \\ Email: adrian.inchauspe@yahoo.com.ar
}

Received 18 February 2016; accepted 24 May 2016; published 27 May 2016

\begin{abstract}
Therapeutic Acupunctural Resonance was first introduced in the academic field in $\mathbf{2 0 1 5}$ and was positively welcomed by colleagues all around the world. However, I have decided to do further research going deeply into new and more precise explanations which no longer refer to the effects of this particular treatment, but to how this technique works in the practice of Chinese Medicine. Being able to understand Therapeutic Acupunctural Resonance implies analyzing a series of phenomena related to historic landmarks of Classical Physics, as well as avant-garde theories within Quantum Physics and its theory of fields.
\end{abstract}

\section{Keywords}

Therapeutic Acupunctural Resonance, Five Elements, Euclid, Pythagoras, Instrumental Mediators, Qi Recondition, QI Sequenced Energetic Re-Configuration

\section{Introduction}

After recently presenting the Therapeutic Acupunctural Resonance during the Congress of Traditional Medicine which took place in Birmingham in 2015, I have found out new discoveries that deeply justify the outcomes of the sessions carried out under this new therapeutic modality [1]. Being able to understand the Therapeutic Acu-

How to cite this paper: Ángellnchauspe, A. (2016) Therapeutic Acupunctural Resonance II: New Discoveries That Justify the Outcomes of This New Therapeutic Modality. Journal of Biosciences and Medicines, 4, 39-45.

http://dx.doi.org/10.4236/jbm.2016.46006 
punctural Resonance implies analyzing a series of phenomena related to historic landmarks of Classical Physics, as well as avant-garde theories of Quantum Physics and its quantum theory of fields.

\section{Material}

\section{Scientific Background of Acupunctural Therapeutic Resonance}

During the Traditional Medicines, I proposed a peculiar parallelism between Euclid's proposition about his Five Regular Polyhedra and the Chinese Theory of the Five Movements, key point for the understanding of the diagnosis and therapeutics of Traditional Chinese Medicine (TCM) [2].

During that 2015 Congress, I reinforced quoted theory about the exact roots that support the parameters and rules of Chinese Medicine, namely Euclid's postulates and Pythagoras' Quint Cycle. Pythagoras was the father of the seven musical notes; of the multiplication table; of the theorem named after him; of the configuration of the first regular pentagon and of the Pythagorean numerology [1].

Additionally, I later found connections between the major pentatonic anhemitonic scale-typical of Chinese music - and the properties that Zou Yen attributed to the emblematic five notes corresponding to the Five Elements coming from Chinese Cosmology.

According to engineer Roberto Tambornino, this way of interpreting the musical representations followed by TCM allow us to introduce substantial changes in its therapeutical methodology that enable the proposition of Instrumental Mediators.

Under this new concept, the musical notes would act in such a way as to cause interaction between the patient's perception and its own nervous system [3].

During the Traditional medicine Congress-2015 I referred to the relation between said Movements and the study of Kinetics - thanks to the sense of impermanence proposed for each of the Elements in the Taoist Philosophy. Thus, it was clearly explained how the Kinetic Theory of Gases and the Theories of States and Conservation of Matter bear a great resemblance with the Generative and Inhibitory Cycles involved in the dynamic interrelation of the Five Elements of Chinese Medicine [1].

It was also presented how we can apply the concept of Tensegrity to biological patterns, in which the function of interconnection of the conjunctive tissue expresses itself in scientific properties derived from Kinetics, such as Mechanical Transduction, Cellular Kinematic Indeterminacy and Dynamic Tensional Integration [1].

\section{Methods}

\subsection{Analysis of the Properties of the Traditional Acupunctural Treatment and/or through Fractal Associated Systems}

There exist numerous studies about the integration of Acupuncture or Auriculotheraphy in Parkinson's disease [4] not only proved to be useful for the control of symptoms [5] such as muscular cramps [6] or tremors [7], as well as for states of anxiety and depression [8]. This therapeutic modality has been duly adopted as a complement of the pharmacologic treatment [9] and proposed for neuro-degenerative symptoms due to their neuroprotector effect [10] among other things because of the inhibition of microglial inflammation [11].

Some time ago, Dr Ullrich Werth described the method of the permanent needle in 2001, which is extensively applied today (over 3000 cases) in his clinic of Valencia [12]. Permanent Auriculotherapy is based on the use of titanium micro implants in the ear's cartilage, thus regulating the dopamine production, highly affected in people with Parkinson's disease. Dr Werth speaks about 95\% of success in people treated with this method [13].

\subsection{Analysis of the Properties of an Instrumental Mediator}

Vygotsky thought that human maturity implies a process of cultural advancement, being man's activity the motor of the process of human development, acting as a bridge between human sciences, Vygotsky tried to find a single solution to the double polarity or alternative between the biological and cultural side [14].

Unlike Piaget, the activity proposed by Vygotsky, is one culturally determined by "mediators" that are used in relation to objects, since the social world is essentially formed by symbolic processes [15].

All the sign systems - the group of phonetic, graphic, tactile, etc. instruments-are considered as a great system of instrumental mediation [14]. Vygotsky believed that man acts over reality adapting to it, transforming 
himself through his “mediators", conceptualized as “tools” (material resources) or "signs” (language, music), which set culturally determined and contextualized interactions in a socially cooperative environment [15].

Some of the following conditions identify an Instrumental Mediator:

- It is a technological support of information that acts as energy transductant.

- It is the carrier of an intentional message in order to cause an effect on the recipient.

- It clarifies the significance of information and the construction of self-awareness.

- It organizes information at pedagogical and therapeutic level as well.

- It implies the application of a kind of knowledge that is intentionally structured.

- It allows to adopt specific symbolisms for learnt knowledge.

- It eases the auto organization of neuro-cognitive learning.

As musical notes are available as instrumental Mediators, we can handle the following items [3]:

- A registry of evaluation ad-hoc (neuro-cognitive tests);

- Quantitative comparable estimations or monitoring of every particular evolution;

- A statistics chart of analysis and average of outcomes/results;

- Analysis of the internal logic of each case in particular;

- Complementary information to the research;

- Neuro-cognitive evaluations comparable in different peoples.

- Optimize the outcomes of conventional acupunctural treatments.

- Improve the operational quality of said treatments for each pathological condition.

- Optimize the changes of technology and/or procedures in Chinese Medicine.

\subsection{Analysis of Kinetic Properties of the Cellular Communication Phenomena Present during Theurapeutic Acupunctural Resonance}

By studies published by Dr. Antonio Gómez Yepes in Coatepec, Mexico, it is considered that the etiology of certain diseases begins in various cellular anomalies: some due to complex mechanisms that form "cascading" cytological phenomena [16].

In his study, Gómez Yepes makes reference to some research entitled: "Search for Quantum and Classical Modes of Information processing in microtubules: Implications for "The Living State" [17] where he convincingly demonstrates that the performance of Cellular Mechanic Transduction through the cytoplasmic microtubules linked to the cytoskeleton allows to register amplified or attenuated vibrations that reach the cellular nucleus to influence the expression of its nuclear genome [2] [16]. Said concepts are similar to the ones expressed by me in Birmingham during 2015 [1].

Cellular Transduction consists in the transmission of data through signals that come from an external origin towards the interior of cells. The vibrations captured by the cytoskeleton give place to extra and intra cellular interactions, responsible for the changes in the transcription of messenger RNA regarding the sequence of its purines and pyrimidines bases. During this process, about 15 to 20 pairs are open making up a "transcription bubble" that is propagated as a "solitary wave" as represented by the nonlinear Klein-Gordon equations and Nash Game Theory [18] [19].

This process could clearly explain the improvement in the making of neurotransmitters that are released during the Therapeutic Acupunctural Resonance, and through them, the restoration of the circardiancrono-biological cycle, which was observed by the Chinese thousands of years ago.

This complex system of Cellular Integrated Tension also determines the existence of quantum mechanisms of communication, as if cellular self-organization needed said information to efficiently complete adaptation, reproduction and cellular evolution [1].

According to Antonio Gómez Yepes, one of the fundamental processes derived from hormonal or cellular mechanical transduction is the greatest activity of Protein kinase $C$ (PKC). It seems as if ischemic injury displaces this enzyme to the cellular membrane, thus activating proteolytic phenomena that lead to a fast tissue degradation [16].

Whether it is under the influence of a magnetic inductive stabilizer or electro-acupuncture, this enzymatic translocation is reversed by the normalization of the PKC levels from the microtubules of the cytoskeleton, turning normal this cellular metabolic capacity.

According to both Gómez Yepes and Mc Clare, it is therefore possible that Mechanical Transduction can al- 
low cells to "capture” energetic vibrations, thus activating "tissue remodeling cascades", as well as neuronal emergency processes. It has been proved that molecules emit specific sound waves/frequencies that "trigger" cellular functional answers, activating the processing of data for intracellular cognition [16].

Jaques Benveniste managed to send virtual molecules from his lab in Clamart (Paris) to the Northwestern University in Chicago only through sound [16].

The evolution of his study through Quantum Mechanics enabled us to identify-from a Physics solid state context — excitations of a quasi-particles collective derived from sound called phonons [20].

To our curiosity, this communication occurs through water molecules that surround the biologic material. It seems as if water is the means that carries and amplifies this communication, process that can be compared to the tuning of a stringed instrument. Let us remember the fact that the human body is formed by $75 \%$ - $80 \%$ of water; and that this manifestation is similar to the concept of soliton [21].

Thus, we have a particular example at hand in order to explain the phenomenon of Therapeutic Acupunctural Resonance through Quantum Mechanics.

\subsection{Analysis of Kinetic, Hydrodynamic and Quantum Properties of Soliton}

\section{Soliton Theory: History and Applications}

Definition of Soliton: A soliton is a "solitary wave" that maintains its shape while travelling in a nonlinear medium.

The phenomenon was first described by the Scottish engineer John Scott Russell. Towards 1834, the government ordered Russell to design a steam boat to sail across the channel that connects Glasgow to Edinburgh.

While studying the motion of boats at varied speed, a strange phenomenon occurred unexpectedly: a boat suddenly stopped, and after moment of inertia, a mass of water rolled forward with great velocity, assuming the form of a large solitary elevation. Russell watched the whole phenomenon along various kilometers, preserving its original form and without weakening. When he first started a systematic experimental research of said phenomenon, he described this "propagation of solitary waves" as a constant situation within the sphere of Hydrodynamics: a balance between non-linear and dispersive effects not until 1965 he called soliton [22].

In Nature there exist other kinds of waves like tsunami that involves maritime underwater currents or pororoca that involves rivers and can be found in the Orinoco and Amazon Rivers [23].

Up until now, optical solitons had practical applications in telecommunications. In 1973, Akira Hasegawa from AT\&T suggested using them in optical networks, improving in this way, their efficiency in transmission; and in 1988 Thierry Georges, from France Telecom, was able to exceed that distance transmitting at a level which was higher than a terabit per second [24].

External physical stimuli-like the ones applied over a neuralgic point in the correct place-can send out signals to other branches of the energetic system and its dynamic structure (Arndt-Schulz Law). In 1924, Ricker predicted the "memory" of the peripheral nervous system, determining that $90 \%$ of the vegetative nervous matter found in the skin. Thus, beyond the embryological origin shared by the teguments and the notochord justifies the action of the cutivisceral reflex during Acupunture [2].

In this way, solitons transmit data through the vibrations in the biological systems, as it happens with nervous impulses (neurosolitons); and they seem to be "holographically" filed in the neurons of the nervous central system after being transported up to there by the peripheral nervous system [25]. This theory would be viable in order to explain the effects and results achieved when integrating Therapeutic Acupunctural Resonanceto Traditional Chinese Medicine.

\section{Discussion}

\section{Notion of the Continuous Limit:}

In the theory of electromagnetic fields, a continuous field can be defined as a group of oscillators coupled among themselves, giving an idea of elastic strength. Macroscopically speaking, the system is defined by the tension in each of its points. The Integrated Tension of those points represents the continuous fields [26].

In a system of coupled oscillators, it would seem that they keep linked by the action of piers [26]. By giving the idea of coupling the oscillators, it will be easier to represent the vibrations of atoms of a solid as a "superposition of its normal frequencies". Considering this phenomenon in a continuous matter, the density or tension of a solid would result in a "superposition of plane waves" of each of its components [27]. 
Thus, each normal or harmonic mode of the group of oscillations would correspond to a certain plane waveof each oscillator-but added to its continuous limit.

In this way we are able to understand the definition of soliton as a solution of equations of nonlinear waves that propagate unaltered in shape [21].

We can observe a great number of clear coincidences between Eastern and Western knowledge: between past and the avant-garde knowledge that leads our present. Therefore, the comparison and explanation of the phenomenon of Therapeutic Acupunctural Resonance through the notion of soliton seems to be adequately correct. Russell's description during his observation is compatible with the nomenclature that identifies the Old Shu points that carry the pathway of energy (Qi) through the main meridians of Acupuncture.

In fact, their sequence clearly illustrates the increase of the Qi channel during its pathway through said points: that is why they are called Bubbling Well, Spring, Stream, River and Sea Points, consequently causing an increase of flow due to their own stimulation [1].

In terms of the Physics of the condensed matter, the description of the atom oscillations of a solid as quasi-particles called phonons, presents theoretical evidence about the possible action of sound over the Therapeutic Acupunctural Resonance channels through Quantum Physics [1] [26].

Using music as an instrumental mediator is an alternative modality of physio-pedagogic as well as therapeutic mediation [3]. The instrumental mediator enables alternative ways that propose new paradigms of selflearning. In this way, these mediators of knowledge establish a complex network that systematically contains, in its structure, all the new information [3].

Vibroacoustic therapy (VAT) showed improvements in all symptoms of Parkinson's disease, including less rigidity and better walking speed with bigger steps and less tremor [28].

A study carried out by the Argentinian Tristán Bekinschtein recently published by the online edition of the scientific magazine "Proceedings" of the National Academy of Sciences, seems to share clear coincidences with the information previously presented in this work [29].

According to Bekinschtein and his team of the French Institute of Medical Research, the cognitive state of a patient that is unable to communicate by himself can be determined by analyzing the electro encephalic register obtained by making him listen to very particular sounds [29].

\section{Conclusions}

At this point, it is important to highlight what was written by Dennis and Joyce Lawson-Wood in their work: "The Five Elements of Acupuncture and Chinese Massage":

"Nothing belongs entirely to one Element excluding the rest. Everything possesses the Five Elements" [30].

The present incidence of Parkinson is really significant: it affects 200 out of 100,000 people and 2 out of 100 among people over 65 [31].

So, will it be possible that Therapeutic Acupunctural Resonance establishes—in a sequential way-an emergent neuro-biological process? According to Francisco Varela:

"Adopting a sequential view of the five aggregates seems to be similar to adopting a sequential view of our brain activity" [32].

In fact, the analysis of the parallelism between the propositions stated by Euclid for his Five Regular Polyhedrons - as formal and mathematical support of the Theory of the Five Movements in Chinese Medicine-appears to suggest it. Moreover, Pythagoras' knowledge and his creation of the harmonic, arithmetic and geometric means, which in turn, supported the Chinese pentatonic scale and the notes corresponding to the Chinese Five Elements [1].

The comparison of the post-puncture propagated sensation or Tchi phenomenon is not enough so as to suggest its compatibility with the soliton, as it may occur during the Neuro-sensitive Transportation [33]. Dr. Edward Lim Chai-si referred to that sensation as "... something that is running" along the meridian being treated. Dr. Lim Chai-si attributed said phenomenon to the activation of neuro-peptidergic type of fibers [1].

Perhaps this pattern achieved by patients while being treated with Therapeutic Acupunctural Resonance may enable them not only to recover lost body functions, but also to re-learn them through a sequenced energetic reconfiguration, empowering their capacity of biological regeneration and recovery [1]. Only in this way will we be able to understand that that Generative Sequence proposed by Zou Yen more than two thousand years ago, encircles within its cycle, a possible "resetting" of the functions lost due to the impairment resulting from degenerative processes. 


\section{References}

[1] Inchauspe, A.A. (2015) Therapeutic Acupunctural Resonance. OMICS Group Traditional Medicine-2015 Congress, Birmingham, 5th August 2015.

[2] Inchauspe, A.A. (2014) Is Traditional Chinese Medicine an Exact Science? OMICS Group Traditional Medicine-2014 Conference and Exhibition on Traditional \& Alternative Medicines, Beijing, 25-26 August 2014.

[3] Folegotto, I. and Tambornino, R. (2001) XVI International Symposium of Informatics in Education (Simposio Internacional de Computación en Educación), México.

[4] Werth, U. (2011). http://comunicacionempresarial.net/UPLOAD/PYM/pym014parkinson_v3.pdf

[5] Carrillo, B. (2015) Parkinson's Disease and Acupuncture.

[6] www.mataifu.org/index.php?arxiu=fitxa_document\&id.contracturas

[7] Tacco Cáceres, V.H. (2012) Control of Parkinson’s Trembling Movements in Acupuncture. (Spanish) https://www.youtube.com/watch?v=_6cf620ckB4

[8] www.parkinsonmadrid.org

[9] http://www.innatia.com/s/c-acupuntura-china/a-beneficios-acupuntura-10172.html

[10] Dominguez Fernandez, N. (2016) Early Beginning Parkinson’s Disease. (Spanish) www.nelyvivirelparkinsonenbaleares.blogspot.com/

[11] www.efisioterapia.net

[12] www.granadahoy.com

[13] Werth, U. (2011). (Spanish) http://www.dsalud.com/index.php?pagina=articulo\&c=1661

[14] http://todoeducativo.over-blog.es/pages/VYGOTSKY-5471284.html

[15] (2011) Instrumental Mediation. (Spanish) http://aldiaconcecar.blogspot.com.ar/2011/06/mediacion-instrumental.html

[16] Gómez Yepes, A. Why Low Frequency Electromagnetic Stimulation Produced by Inductive Magnetic Stimulator (EIMA in Spanish) Works Well in the Treatment of Various Diseases Including Autoimmune Ones? Coatepec, México, 2-7. http://www.terapiasmetabolicas.com/eima porquefunciona/

[17] Hameroff, S. and Tuszinski, J. (2002) Search for Quantum and Classical Modes of Information Processing in Microtubules, Implications for “The Living-State”. Energy and Information Transfer in Biological Systems, 32-62.

[18] Rodríguez Quintero, N. (2005) Simple DNA Mathemaicalmodels: Uses and Limitations (Modelos matemáticos sencillos del ADN: su utilidad y limitaciones). Summer Course of Biology, International University of Andalucía, Antonio Machado, Baeza, Jaen, Spain, 8-12 August 2005.

[19] Yakushevich, L. (2004) Nonlinear Physics of DNA. Wiley WCH.

[20] Wikipedia. Quantum Theory of Fields (“Teoría-Cuántica-de-Campos”). 2-11.

[21] Álvarez-Nodarse, R. (2005) Mathemaicalmodels in Biology (Modelos matemáticos en Biología. Goals (“Objetivos”). International University of Andalucía, Antonio Machado, Baeza, Jaen, Spain, 8-12 August 2005, 2-6. http://euler.us.es/ renato/adn3/adn//

[22] Naumkin, P. (2008) What Is a Soliton? UNAM Bulletin, $N^{\circ}-19$, Mathematical Institute, Morelia-Campus, Autonomus University of Mexico, 1-4.

[23] Mucha, M. (2015) The Most Large Wave Could Be "Surfed” in the Amazon River (La ola más larga se "surfea” en el Amazonas).

[24] Hasegawa, A. and Tappert, F. (1973) Transmission of Stationary Nonlinear Optical Pulses in Dispersive Dielectric Fibers. I. Anomalous Dispersion. Applied Physics Letters, 23, 142-144,

[25] Nagasawa, T. and Nishida, Y. (1981) Experiments on the Ion-Acoustic Cylindrical Solitons. Plasma Physics, 23.

[26] Wikipedia. Quantum Theory of Fields (“Teoría-Cuántica-de-Campos”). 3011

[27] Herrera-Aguilar, A. and Téllez-Vázquez, J.O. () Solitones en la teoría Einstein-Maxwell-dilatón-axión. Mexican Journal of Physics, 51, 549-557.

[28] www.medicalpress.es

[29] http://www.lanacion.com.ar/nota.asp?nota id=1093777\&pid=5751842\&toi=6275

[30] Lawson-Wood, D.J. (1979) The Five Elements of Acupuncture and Chinese Massage, Chap. IV. Colección Medicina y Natura, Primera edición Visión Libros, Barcelona, 41.

[31] (2012) Parkinson's disease according to Chinese Medicine http://practitionerregister.blogspot.com.ar/2012/01/enfermedad-de-parkinson-segun-medicina.html 
[32] Varela, F., Thompson, E. and Rosch, E. (1992) The-Emodied-Mind: Cognitie Science and Human Experience. Ed. Gedisa, Barcelona, 115.

[33] Russell, J.S. (1844) Report on Waves. 14th Meeting of the British Association for the Advancement of Science,. 